\author{
L. SPANI MOLELLA \\ R.-H. RINKLEFF \\ G. KÜHN \\ K. DANZMANN
}

\section{Giant Kerr effect in closed degenerate two-level transitions}

Albert-Einstein-Institut, Max-Planck-Institut für Gravitationsphysik and Institut für Gravitationsphysik, Gottfried-Wilhelm-Leibniz-Universität Hannover, Callinstraße 38, 30167 Hannover, Germany

\section{Received: 14 September 2007}

Published online: 14 November 2007 • (C) Springer-Verlag 2007

ABSTRACT Extremely large optical Kerr non-linearities $\left(n_{2}\right)$ and $\left(n_{4}\right)$ were measured in two closed degenerate two-level transitions in the caesium $D_{2}$ line when a probe and a coupling laser simultaneously probed and coupled the hyperfine structure in an atomic beam. The magnitude of the measured non-linearities in both transitions were found to be a function of the coupling laser intensity and of the probe detuning from the two-photon resonance. In a figure of merit, the ratio between the non-unitarian part of the refractive index and the absorption coefficient measured in conditions of electromagnetically induced transparency showed a maximum value for a probe detuning of $160 \mathrm{kHz}$ from the two-photon resonance and resulted in being $10^{5}$ times larger than in the absence of the coupling laser field.

PACS 32.70.Jz; 42.50.Gy; 42.65-k

\section{1}

\section{Introduction}

The Kerr effect is a non-linear effect which couples the changes in the refractive index of a material to the intensity of an applied electric field. Kerr non-linearities correspond to the refractive part of the third order susceptibility in optical media and play a crucial role in non-linear optics for the generation of optical solitons, frequency conversion, polarisation phase gates, quantum logic, ... (see [1] and references therein). In recent years various schemes for generating large Kerr non-linearities were proposed and implemented: optical fibres fabricated with novel glass materials [2], microcavities at room temperature [3], thin films [4], and atomic coherence [5].

In this publication, partly stimulated by the current research into gravitational-waves, we want to transfer the information acquired in studying the dispersion of closed two-level systems [6] and the associated giant Kerr effect to concepts which are closer to those used in the development of future gravitational wave detectors. It is expected that the coming generations of earth-based interferometers for the detection of gravitational waves will - at some point - be limited by quantum noise. The insertion of media exhibiting a non-negligible Kerr

Fax: +49 (0)511 762 2784, E-mail: Luca.Spani.Molella@aei.mpg.de effect under the action of the applied electric field in the interferometer cavities is one of the methods suggested to beat the standard quantum limit and thus to improve the performance of future detectors [7]. Furthermore, some of us have experimentally demonstrated that a transparent Kerr medium inside a cavity drastically changes the properties of the cavity [8].

It has been demonstrated that in the proximity of a twophoton resonance responsible for electromagnetically induced absorption (EIA $[9,10])$ or electromagnetically induced transparency (EIT $[11,12])$ within the caesium $D_{2}$ line, giant Kerr non-linearities can be measured [13]. These results have been obtained in a caesium cell, where the maximum linear absorption did not exceed 60\%. However, a characterisation of the Kerr effect and of the corresponding medium absorption is needed to estimate the potential efficiency of such a non-linear medium inside a large interferometer. In this way one can find an optimal operating point to drive the non-linear medium.

In this paper we want to determine and compare the performance of various atomic transitions as Kerr media. To this end, we analysed the absorption coefficient, the refractive index, and the ratio between the non-unitarian component of the refractive index and the absorption coefficient, which we called figure of merit (FOM). We present a comparison of the results obtained in the two closed transitions of the $D_{2}$ line of caesium. The measurement were performed on an atomic beam. One of the transitions was characterised by EIT, the other by EIA. These transitions had different angular momenta both in the ground and in the upper state, being $6 s^{2} S_{1 / 2}$, $F=3$ to $6 p{ }^{2} P_{3 / 2}, F^{\prime}=2$ and $6 s^{2} S_{1 / 2}, F=4$ to $6 p{ }^{2} P_{3 / 2}$, $F^{\prime}=5$.

2

Experimental setup

To measure the refraction and absorption of the optical transitions we used a heterodyne interferometer consisting of three laser beams. The experimental setup is shown in Fig. 1. The details of this experimental arrangement have been described in a previous publication [14]. In short, all optical beams in the experiment - a coupling, a probe, and a reference laser beam - were generated by single mode diode lasers. These were self-injection locked lasers, which were passively stabilised with an external optical feedback from a cavity. The frequency of the coupling laser was actively locked to the in- 


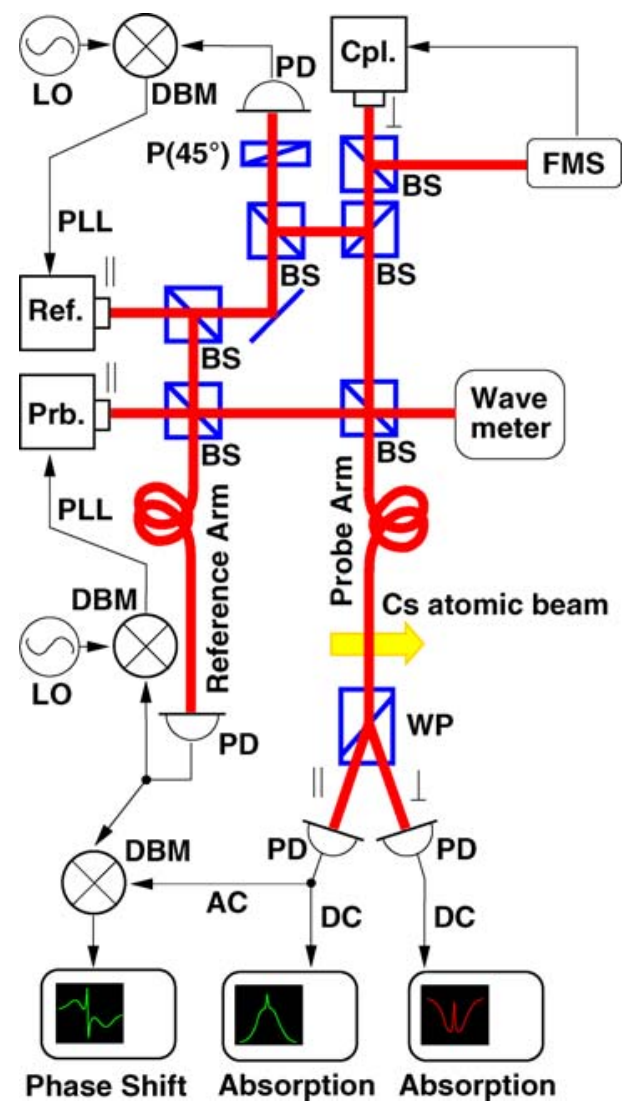

FIGURE 1 Experimental setup: Cpl., Ref., Prb.: coupling, reference, and probe laser; LO: local oscillator; PD: photodiode; DBM: double balanced mixer; BS: beam splitter; WP: Wollaston prism; $\mathrm{P}\left(45^{\circ}\right)$ : polariser oriented at $45^{\circ}$ with respect to the impinging laser beam; FMS: frequency modulation spectroscopy; PLL: phase-locked loop

vestigated transition via frequency modulation spectroscopy. The reference laser was phase-locked with a tunable offset of $\delta+1 \mathrm{GHz}$ to the coupling laser and the probe laser was phase-locked with a fixed offset of $\Delta v_{\text {off }}=1 \mathrm{GHz}$ to the reference laser. The differences of the frequencies were precisely controlled by radio frequency generators. The probe and reference lasers shared the same linear polarisation whereas the coupling laser was linearly polarised with a polarisation orthogonal to that of the probe.

The coupling and probe lasers simultaneously drove the selected transition. We assumed no influence of the reference field on the atomic sample, because of its large frequency off- set from the driven atomic transition frequency (about $1 \mathrm{GHz}$ ). The laser fields were superposed at the beam splitters, where the reference and probe arm of the heterodyne interferometer were generated. All three fields were guided through singlemode fibres to achieve a good mode matching.

The laser beams in the probe arm crossed the caesium atomic beam orthogonally to its propagation direction and were subsequently separated by a Wollaston prism. This configuration yielded Doppler-free spectra. In the interaction zone the waist of the laser beam was $2.2 \mathrm{~mm}$ and the diameter of the atomic beam $5 \mathrm{~mm}$. The atomic density was $4 \times 10^{-15} \mathrm{~m}^{-3}$. Three coils in the three spatial directions suppressed the environmental magnetic field in the interaction region below $0.5 \mu \mathrm{T}$ so that degeneration in the Zeeman structure could be assumed.

The interference of the reference-laser field with the probe-laser field on the photo-detectors at the end of the probe and reference arms generated beat signals. These oscillated at a frequency corresponding to the difference in frequency between the fields. While the amplitude and phase in the reference arm remained constant, the phase and amplitude in the probe arm depended on the absorption and dispersion of the coupling field in the sample. The absorption was directly determined by measuring the dc photo-current of the photoreceiver:

$I_{\mathrm{dc}}(\delta) \propto\left(E_{0}^{\text {probe }}\right)^{2} \mathrm{e}^{-2 \alpha(\delta) l}+\left(E_{0}^{\text {ref }}\right)^{2}$.

$\alpha(\delta)$ was the absorption coefficient of the probe laser and $l$ the interaction length. The phase shift $\mathrm{d} \varphi$ induced by the medium was obtained from the mixer output signal:

$I_{\text {mix }}(\delta) \propto\left(E_{0}^{\text {probe }} E_{0}^{\text {ref }}\right)^{2} \mathrm{e}^{-\alpha(\delta) l} \sin (\mathrm{d} \varphi(\delta)-\Phi)$

with $\Phi=2 \pi \Delta v_{\text {off }}\left(L_{\text {probe }}-L_{\text {ref }}\right) / c$, where $L_{\text {probe/ref }}$ were the arm lengths of the interferometer. By an appropriate choice of the reference frequency it was possible to adjust $\Phi$ to zero.

\section{Experimental results}

Typical probe absorption signals measured in the transitions $6 s{ }^{2} S_{1 / 2}, F=3$ to $6 p{ }^{2} P_{3 / 2}, F^{\prime}=2$ and $6 s{ }^{2} S_{1 / 2}$, $F=4$ to $6 p{ }^{2} P_{3 / 2}, F^{\prime}=5$ are plotted in Fig. 2. At the two photon resonances, the first transition showed enhanced transparency (EIT) and the second enhanced absorption (EIA).

The results of the phase-shift measurements in the transition $6 s^{2} S_{1 / 2}, F=4$ to $6 p^{2} P_{3 / 2}, F^{\prime}=5$ at various couplinglaser intensities are presented in Fig. 3. These results were
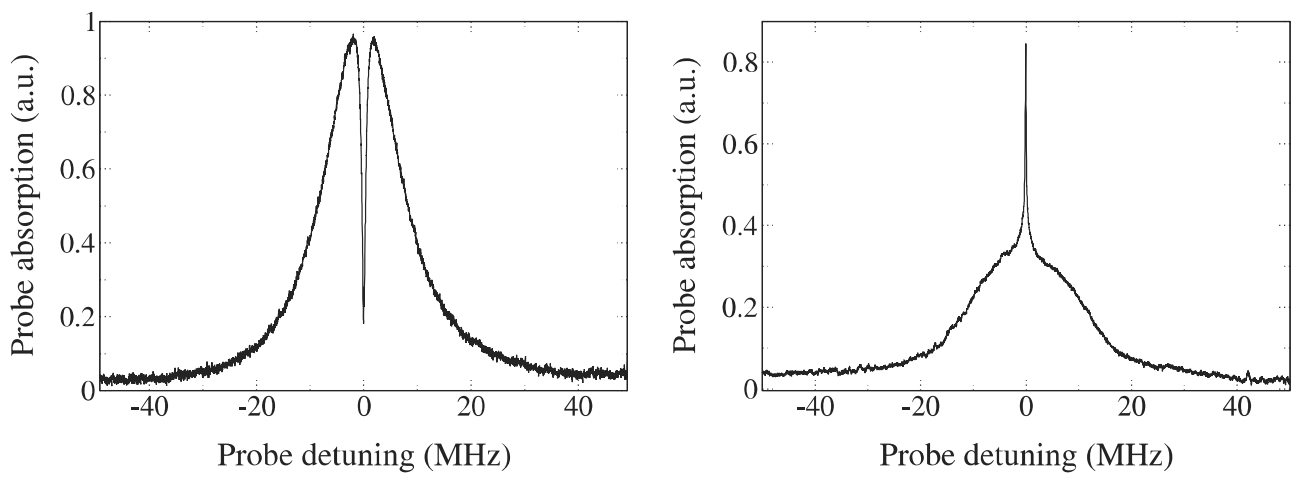

FIGURE 2 Typical probe-laser EIT and EIA spectra measured on the $F=3$ to $F^{\prime}=2$ (left) and the $F=4$ to $F^{\prime}=5$ (right) transition 


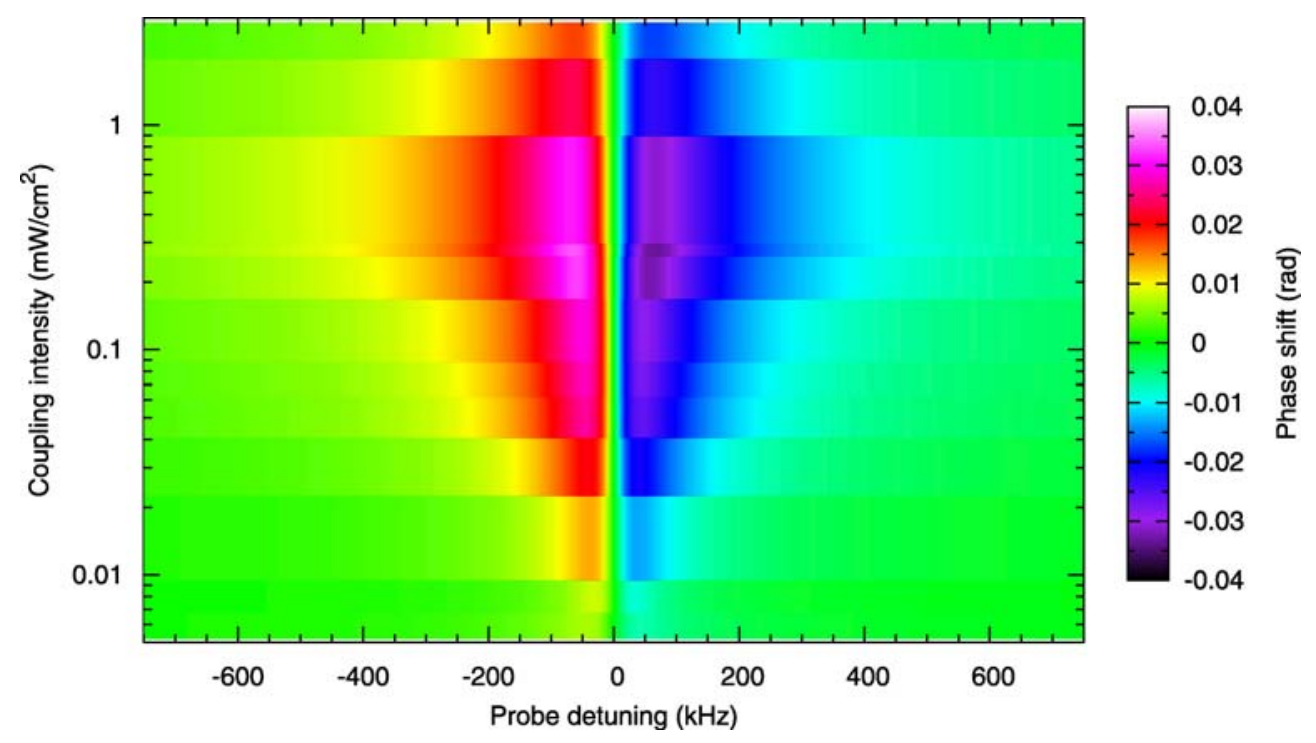

FIGURE 3 Probe-laser phase shift for various probe laser detunings from the EIA two-photon resonance transition and various coupling laser intensities

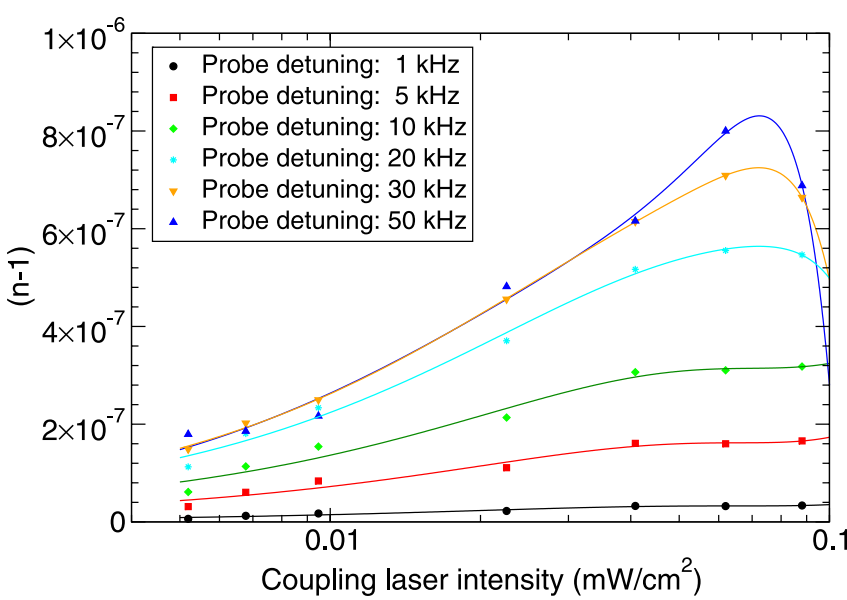

FIGURE 4 Probe refractive index difference from unity as a function of the coupling-laser intensity. Each curve corresponds to a specific probefrequency offset

obtained by fitting the probe-dispersion spectra to a theoretical dispersion profile, thus obtaining the probe-laser phase shift as a two-dimensional function of the probe laser detuning and of the applied coupling laser intensity. From these results it was possible to extract the refractive index of caesium as a function of the coupling-laser intensity for various detunings of the probe laser from the EIA two-photon resonance (see Fig. 4).

It can be shown that the refractive index of a Kerr medium can be expressed as a function of the intensity $I_{\text {coupl }}$ of the applied field (here the coupling field) in the form

$n\left(I_{\text {coupl }}\right)-1=n_{0}+n_{2} I_{\text {coupl }}+n_{4} I_{\text {coupl }}^{2}+\ldots$

where $n_{0}$ is the linear part of the refractive index while $n_{2}$ and $n_{4}$ are the first two non-linear Kerr coefficients. Through a polynomial fit (based on the previous expansion) of the values of the refractive index obtained at various probe detunings from the two-photon resonance, we yielded information on the linear and non-linear components of the refractive index. These results are plotted in Fig. 5. The fitting errors for $n_{2}$ and $n_{4}$ were smaller than $20 \%$. These

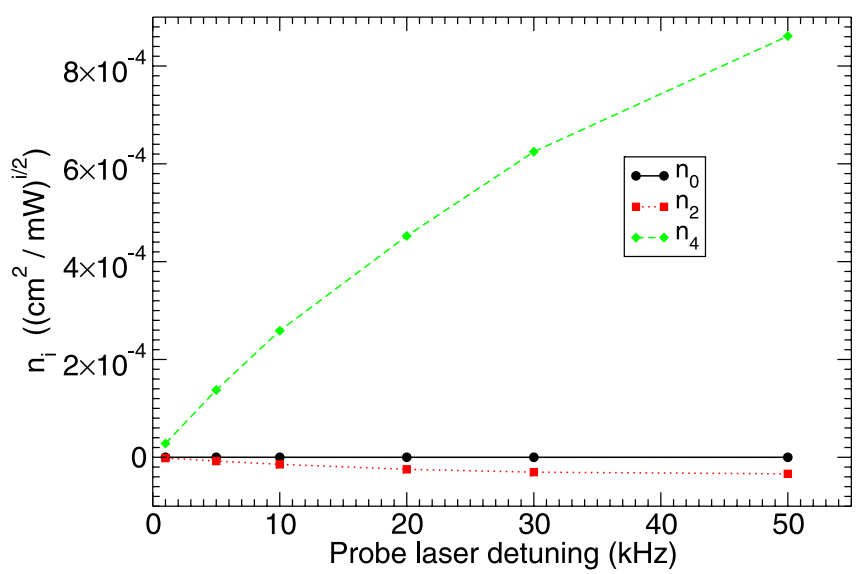

FIGURE 5 Kerr coefficients at various probe detunings from the EIA twophoton resonance

results can be compared with those presented in [13], where, using a cell and in different experimental conditions (atomic density not comparable with that of the atomic beam, coupling laser powers up to $6 \mathrm{~mW}$ ), the Kerr coefficient was determined to be $n_{2}=-(0.74 \pm 0.07) \times 10^{-6} \mathrm{~cm}^{2} \mathrm{~mW}^{-1}$ at a probe-laser detuning of $44 \mathrm{kHz}$. In the present case, at $50 \mathrm{kHz}$ detuning, we measured non-linear Kerr coefficients $n_{2}=-(34 \pm 7) \times 10^{-6} \mathrm{~cm}^{2} \mathrm{~mW}^{-1}$ and $n_{4}=-(0.86 \pm$ $0.16) \times 10^{-3} \mathrm{~cm}^{4} \mathrm{~mW}^{-2}$.

The results for the Kerr coefficients can be properly understood only if one compares the values obtained here for $n_{2}$ with the typical values for an ordinary material, such as glass. In glass one has $n_{2} \simeq 10^{-20} \mathrm{~cm}^{2} \mathrm{~mW}^{-1}$, whereas in the case of caesium the value measured here was about $10^{-5} \mathrm{~cm}^{2} \mathrm{~mW}^{-1}$. The improvement obtained with atomic caesium is still remarkable even if compared with typical Kerr coefficients of media commonly used to generate Kerr nonlinearities, such as GaAs, where a value of $\left|n_{2}\right| \simeq 2.7 \times$ $10^{-16} \mathrm{~cm}^{2} \mathrm{~mW}^{-1}$ is reported at a wavelength of $1064 \mathrm{~nm}$ [15], or polydiacetylene, with an $n_{2}$ Kerr coefficient of $\left|n_{2}\right| \simeq$ $3 \times 10^{-11} \mathrm{~cm}^{2} \mathrm{~mW}^{-1}$ [15]. Under conditions of extreme absorption, at a wavelength of $852 \mathrm{~nm}$, the value $\left|n_{2}\right| \simeq 2 \times$ $10^{-7} \mathrm{~cm}^{2} \mathrm{~mW}^{-1}$ is reported [16] for the Kerr coefficient of 
GaAs. However, at this wavelength, the absorption coefficient of GaAs has the value of $\alpha \simeq 10^{4} \mathrm{~cm}^{-1}$ while the absorption coefficient for EIA measured in our experiment at the same frequency was of the order of $0.1 \mathrm{~cm}^{-1}$.

Even if the absorption coefficient is small, the Kerr medium in the presence of electromagnetically induced absorption is characterised by an enhanced absorption with respect to a one-photon two-level transition. This might make it unattractive for implementation in a cavity in a gravitationalwave interferometer.

As the EIT signals measured on the $F=3 \rightarrow F^{\prime}=2$ closed transition were taken in the presence of drastically reduced absorption and had roughly the same width as the EIA signals on the closed $F=4 \rightarrow F^{\prime}=5$ transition, we also calculated the Kerr coefficients in this case. The results of the phase shift are shown in Fig. 6. The presence of an EIT dip caused the phase shift to be zero at two further points besides the two-photon resonance. Moreover, the sign and the slope of the phase shift as a function of the probe detuning are opposite to the signal without coupling laser in the vicinity of the EIT-signal. As the dispersion signals are characterised by a broader negative dispersion profile with a central positive dispersion profile at the two-photon resonance, we present two different plots which highlight the two dispersive features.

With a procedure similar to that adopted for the probe laser acting on the $F=4 \rightarrow F^{\prime}=5$ transition, we extracted the probe refractive index from the phase shift at various probe detunings and, finally, the Kerr coefficients (Fig. 7). The extremal values $n_{2}=(0.13 \pm 0.02) \times 10^{-6} \mathrm{~cm}^{2} \mathrm{~mW}^{-1}$ and $n_{4}=$ $(-8.8 \pm 1.7) \times 10^{-9} \mathrm{~cm}^{4} \mathrm{~mW}^{-2}$ at a probe detuning of 880 $\mathrm{kHz}$ can be compared with the values reported in [13] $n_{2}=$ $(0.2 \pm 0.02) \times 10^{-6} \mathrm{~cm}^{2} \mathrm{~mW}^{-1}$ and $n_{4}=(-0.02 \pm 0.01) \times$ $10^{-6} \mathrm{~cm}^{4} \mathrm{~mW}^{-2}$ at a probe detuning of $350 \mathrm{kHz}$. Even if the Kerr coefficients obtained in the presence of EIT were about two orders of magnitude smaller than those measured in the
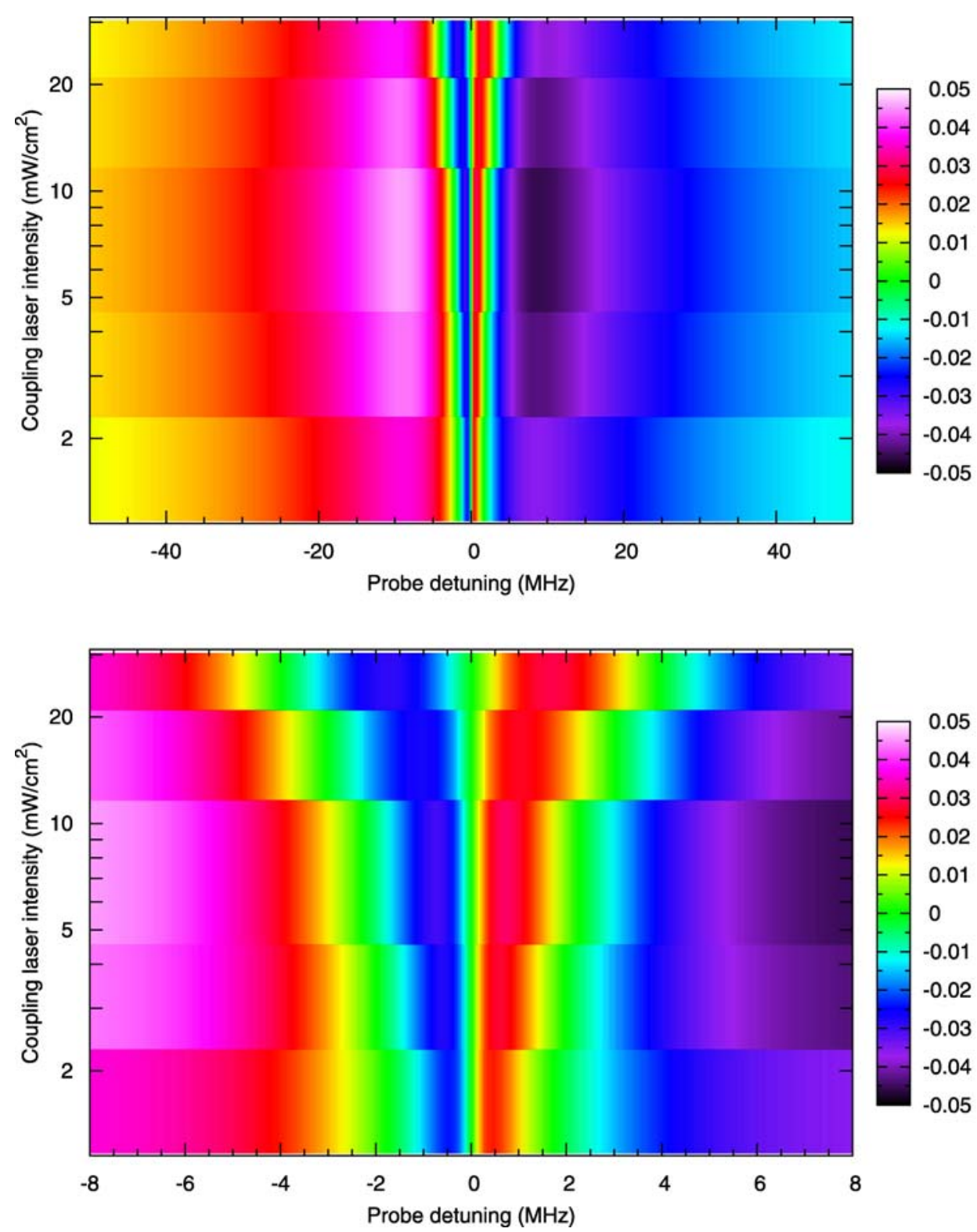

FIGURE 6 Probe phase shift as a function of the probe laser detuning from the EIT two-photon resonance for various coupling laser intensities. The figure on the bottom zooms the central feature of the figure on the top 


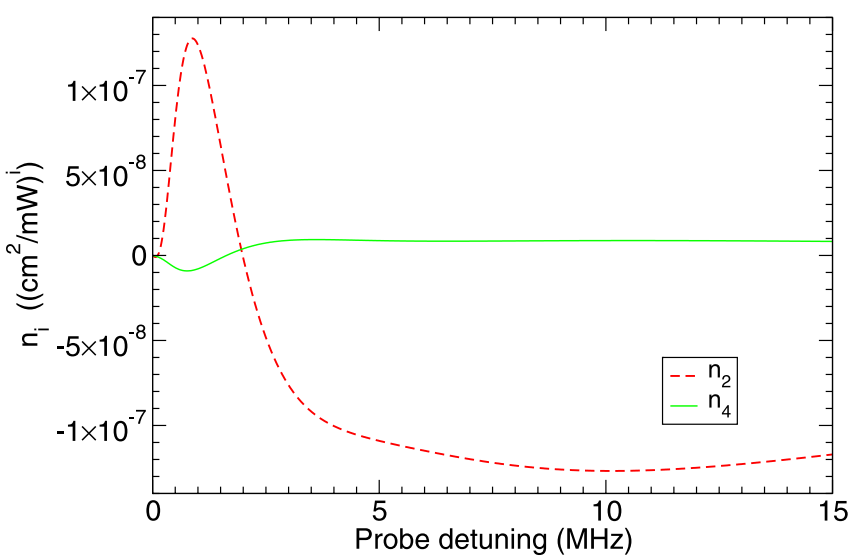

FIGURE 7 Non-linear Kerr coefficients at various probe laser detunings from the EIT two-photon resonance frequency

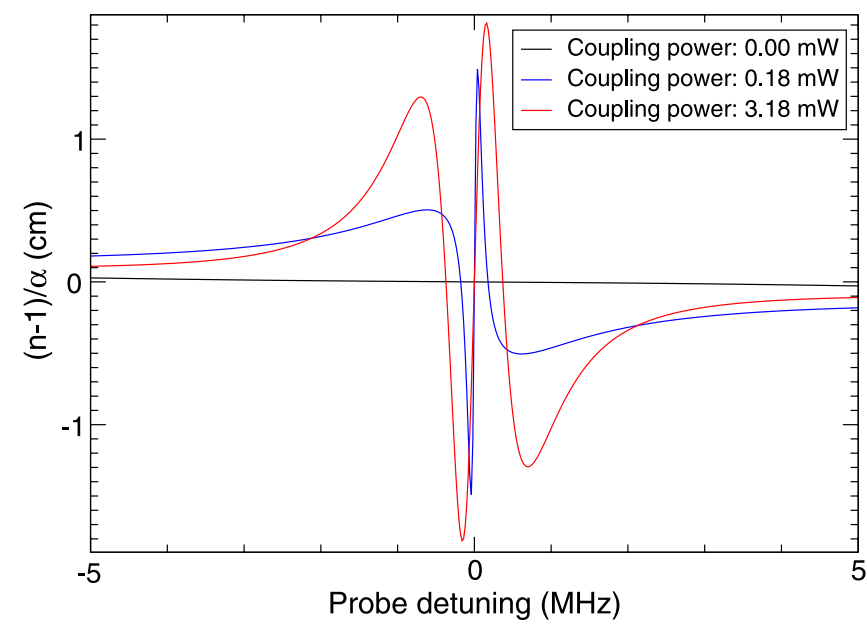

FIGURE 8 Figure of merit (FOM): ratio between the non unitarian component of the refractive index and the absorption coefficient of the medium in the presence or absence of the coupling laser

presence of EIA, they were obtained for a medium which could be considered transparent for all practical purposes. Due to its almost lossless transmissivity an EIT-medium constitutes an excellent candidate for the realisation of a Kerr interferometer.

Because in the EIT case the medium was transparent, it was of particular interest to calculate a figure of merit which connected the dispersion at a fixed detuning with the induced transparency at the same detuning. In this way it was possible to locate the optimal working point for the medium, which was determined by a compromise between the transparency level and the induced phase shift. The optimal working point changed when the coupling laser intensity was varied. Two figures of merit obtained at two different coupling laser powers and the same probe laser power are shown in Fig. 8 together with the figure of merit of a probe laser in the absence of the coupling laser.
For a coupling power of $3.18 \mathrm{~mW}$, corresponding to the best working point for a probe laser power of $0.30 \mathrm{~mW}$ (with a beam waist of $2.2 \mathrm{~mm}$ ), the maximum figure of merit was obtained for a probe detuning of $160 \mathrm{kHz}$ from the two-photon resonance. At this maximum, the figure of merit was $10^{5}$ times larger than in the absence of the coupling laser (i.e. in the presence of a simple one-photon transition for the probe laser). For a coupling power larger than $3.18 \mathrm{~mW}$ the maximum of the figure of merit decreased. The corresponding frequency simultaneously shifted to higher frequency detunings. This behaviour could be ascribed to the lower steepness of the probe dispersion curve at high coupling powers which was accompanied by power-broadening of the line width.

\section{4}

\section{Conclusions}

We have measured the phase shift and absorption of closed degenerate two-level transitions in caesium. From these measurements we extrapolated higher order Kerr nonlinearities. In the EIT medium losses of the probe laser intensity are almost completely suppressed in the vicinity of the two-photon resonance. Therefore, it is in principle possible to use this medium inside a cavity in a gravitational interferometer to beat the standard quantum limit.

ACKNOWLEDGEMENTS We thank the German Research Foundation (Deutsche Forschungsgemeinschaft) for the support to this research through the Collaborative Research Centre SFB 407.

\section{REFERENCES}

1 M. Fleischhauer, A. Imamoglu, J.P. Marangos, Rev. Mod. Phys. 77, 633 (2005)

2 J.H. Lee, T. Taremura, K. Kikuchi, T. Nagashima, T. Hasegawa, S. Ohara, N. Sugimoto, Opt. Lett. 39, 1267 (2005)

3 H. Rokhsari, K.J. Vahala, Opt. Lett. 30, 427 (2005)

4 E. Fazio, A. Passaseo, M. Alonzo, A. Belardini, C. Sibilia, M.C. Larciprete, M. Bertolotti, J. Opt. A 9, L3 (2007)

5 H. Schmidt, A. Imomoglu, Opt. Lett. 21, 1936 (1996)

6 L. Spani Molella, R.-H. Rinkleff, K. Danzmann, Phys. Rev. A 72, 041 802(R) (2005)

7 H. Rehbein, J. Harms, R. Schnabel, K. Danzmann, Phys. Rev. Lett. 95, 193001 (2005)

8 G. Müller, M. Müller, A. Wicht, R.-H. Rinkleff, K. Danzmann, Phys. Rev. A 56, 2385 (1997)

9 A.M. Aklushin, S. Barreiro, A. Lezama, Phys. Rev. A 57, 2996 (1998)

10 C. Goren, A.D. Wilson-Gordon, M. Rosenbluh, H. Friedmann, Phys. Rev. A 69, 053818 (2004)

11 S.E. Harris, Phys. Today 50, 36 (1997)

12 E. Arimondo, in Progress in Optics, ed. by E. Wolf (Elsevier, Amsterdam, 1996), Vol. 35, p. 257

13 A.M. Akulshin, A.I. Sidorov, R.J. McLean, P. Hannaford, J. Opt. B 6, 491 (2004)

14 L. Spani Molella, R.-H. Rinkleff, K. Danzmann, Spectrochim. Acta A 63, 987 (2006)

15 M.B. Flynn, L. O’Faolain, T.F. Krauss, J. Opt. Soc. Am. B 22, 792 (2005)

16 D.A.B. Miller, D.S. Chemia, D.J. Eilenberger, P.W. Smith, A.C. Gossard, W. Wiegemann, Appl. Phys. Lett. 42, 925 (1983) 